\title{
Induced currents, frozen charges and the quantum Hall effect breakdown
}

\author{
K.V. Kavokin $\dagger$, M.E. Portnoi $\ddagger$, A.J. Matthews $\dagger$, J. Gething $\dagger$, A. Usher $\dagger$ \\ D.A. Ritchie§, and M.Y. Simmons§ \\ $\dagger$ School of Physics, University of Exeter, Exeter EX4 4QL, United Kingdom \\ $\ddagger$ A. F. Ioffe Physico-Technical Institute, 194021 St Petersburg, Russia \\ $\S$ Cavendish Laboratory, Cambridge CB3 0HE, United Kingdom
}

\begin{abstract}
Puzzling results obtained from torque magnetometry in the quantum Hall effect (QHE) regime are presented, and a theory is proposed for their explanation. Magnetic moment saturation, which is usually attributed to the QHE breakdown, is shown to be related to the charge redistribution across the sample.
\end{abstract}

Since the discovery of the quantum Hall effect (QHE), experiments using strong magnetic fields and low temperatures have continued to give new information on the physics of two-dimensional electron systems (2DESs). However, much remains to be understood. One of the most puzzling phenomena is the so-called QHE breakdown a complex of nonlinear effects arising when high-density currents are passed through a 2DES. Many theoretical models have been proposed to explain it [1], although no universal agreement has yet been reached. Among the most fundamental reasons for QHE breakdown at some current density in any 2DES, the quantum inter-Landaulevel scattering (QUILLS) [2] deserves mention. The QUILLS process consists in tunneling of electrons between adjacent Landau levels (LLs), which becomes possible when the in-plane electric field reaches values comparable to $\hbar \omega_{c} / l_{H}$, where $\hbar \omega_{c}$ is the cyclotron energy, and $l_{H}$ is the magnetic length. Much experimental effort has been spent on detecting QUILLS, but no reliable results have been so far obtained. There are many indications that the QHE breakdown observed in traditional QHE experiments is strongly affected by contacts [1]. To avoid contact effects, it was proposed to induce currents in a 2DES by sweeping magnetic field and to detect these currents with a high-sensitivity torque magnetometer [3]. By using this technique, an effect was observed which was qualitatively consistent with the QHE breakdown, but demonstrated many unusual properties that could not be explained within existing theories.

Here we analyse the experimental data from Ref. 3, as well as new data obtained with the same method, employing a theoretical model based upon an idea proposed by Dyakonov [4], which we have developed to account for the specific features of the contactless experiment.

The basic features of the experiment are as follows (full details are given in Ref. 3). A sample (typical size $\sim 1 \mathrm{~cm}$ ) is suspended on the filament of a torque magnetometer and placed into a superconducting solenoid. The magnetic field is then swept at a constant rate. The component of the magnetic field perpendicular to the sample induces a circulating electric field that drives eddy currents in the 2DES. The currents create a magnetic moment which is detected by the magnetometer. Sharp peaks in magnetic field dependence of the induced magnetisation are observed at integer filling 
factors, $\nu$ (Fig.1). This is a manifestation of vanishing longitudinal resistivity, $\rho_{x x}$, in the integer QHE regime. The saturation of the peak height with increasing the sweep rate is observed in all the samples. This feature is associated with the QHE breakdown: the circulating electric field fails to induce currents larger than a certain value. The saturation value of the magnetic moment, $M_{S}$, is the main quantitative characteristic of the observed effect. The experiment reveals a few astounding features which can not be explained by any previous theory of the QHE breakdown:

1) $M_{S}$ can reach much higher values in 'dirty', more disordered, samples with lower mobility of charge carriers at zero magnetic field.

2) Induced currents can be detected only at fairly low temperatures (typically $T<1.5 \mathrm{~K})$, well below the cyclotron energy.

3) In 'dirty' samples, the temperature dependence of $M_{S}$ is best fitted by a descending straight line (see Fig. 1, inset); in some cases, a 'shelf' at low temperatures is observed.

4) All these features are apparently insensitive to the polarity of charge carriers and the chemical composition of the structure. Similar behaviour has been observed for electrons in a GaAs/(Al,Ga)As heterostructure and for holes in a SiGe heterostructure.

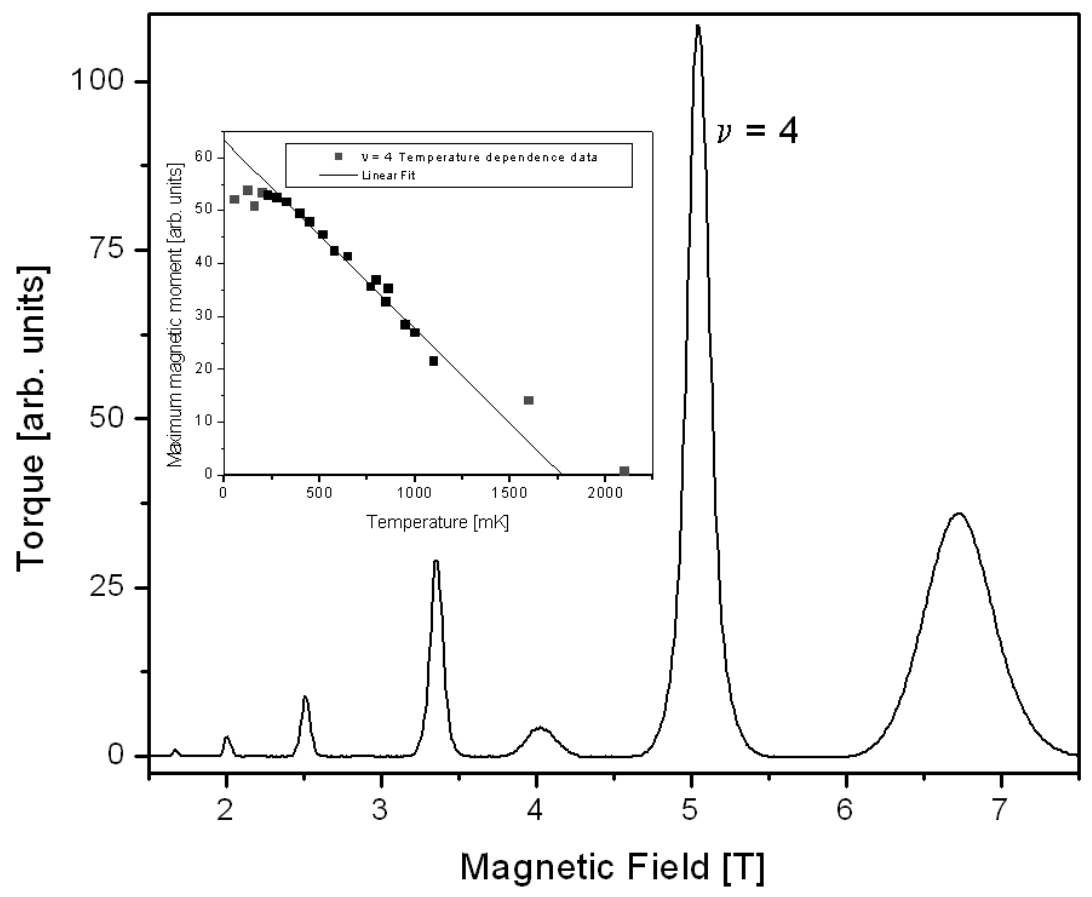

Figure 1: Induced magnetic moment vs magnetic field at a constant sweep rate; Inset: saturation value of the induced magnetic moment vs temperature for a 'dirty' sample

Our theoretical model considers a disc-shaped sample with the radius $R$. The induced magnetic moment $M$ can be written as an integral over the sample of the 
tangential component of the current density, $j_{\varphi}(r)$ :

$$
M=\frac{\pi}{c} \int_{0}^{R} j_{\varphi}(r) r^{2} d r=\frac{\pi}{c} \int_{0}^{R} \sigma_{x y} E_{r}(r) r^{2} d r,
$$

where $E_{r}$ is the radial component of the in-plane electric field. Here we used the fact that in the QHE regime $j_{\varphi} \approx \sigma_{x y} E_{r}$.

Thus, to calculate the magnetic moment one has to find the radial electric field throughout the sample. This electric field is created by charges which are redistributed within the sample in such a way that the sample as a whole remains electrically neutral. As noticed by Dyakonov [4], in two-dimensional systems these charges cannot be concentrated at the edges, but must be distributed over the plane. In contactless experiments with disc-shaped samples, the validity of this statement is evident: there are simply no edge states in the centre of the sample, where some excess charge (positive or negative) should be placed. The excess or deficit, $\Delta n$, of charge carriers in the 2DES plane results in shifting the Fermi-level, $\varepsilon_{F}$, from its initial position $(\varepsilon=0)$. The probability of thermal activation of a charge carrier (an electron to the lowest empty level, or a hole to the uppermost filled level) is proportional to $\exp \left[-\left(\varepsilon_{0} / 2-\varepsilon_{F}(\Delta n)\right) / k_{B} T\right]+\exp \left[-\left(\varepsilon_{0} / 2+\varepsilon_{F}(\Delta n)\right) / k_{B} T\right]$ (where $\varepsilon_{0}$ is the energy distance between the uppermost filled and the lowest empty electron levels in an idealised 2DES). As long as $|\Delta n|$ is small, this is a very small number. With an increase of the absolute value of $\Delta n$, one of the exponentials increases and the other decreases, so that it can be neglected. This results in an exponential dependence of the longitudinal conductivity, $\sigma_{x x}$, on $\left|\varepsilon_{F}(\Delta n)\right|$. As a consequence, $\sigma_{x x}$ should demonstrate a threshold behaviour: there is no mobile charge until the exponent is less than some critical value; above this value, the conductivity is high enough to provide charge relaxation, so that the radial field, $E_{r}$, can not be sustained. The threshold condition can be written as:

$$
\exp \left(-\frac{\varepsilon_{0} / 2-\left|\varepsilon_{F}(\Delta n)\right|}{k_{B} T}\right)=C,
$$

where $C$ is a small number. Using Eq. (2) we find the threshold value of $|\Delta n|$ as

$$
\Delta n_{c}(T)=\int_{0}^{\varepsilon_{F}} \rho(\varepsilon) d \varepsilon=\int_{0}^{\varepsilon_{0} / 2+k_{B} T \ln C} \rho(\varepsilon) d \varepsilon,
$$

where $\rho(\varepsilon)$ is the density of localised electron states. This result allows us to construct the electric field distribution in the sample which would correspond to saturation of the magnetic moment in the QHE regime. Indeed, the threshold behavior of conductivity allows two situations: (i) $|\Delta n|<\Delta n_{c}(T)$ at any $E_{r}$ or (ii) $|\Delta n|>\Delta n_{c}(T)$ at $E_{r}=0$. As we are interested in charge distributions that would provide maximum possible $E_{r}$, we construct it on the basis of condition (i) using, however, condition (ii) in small parts of the sample to provide self-consistency of the solution. The obvious first approximation for the charge distribution which gives the maximum possible value of $M$ is the following: $+e \Delta n_{c}(T)$ at $r<R / \sqrt{2}$ and $-e \Delta n_{c}(T)$ at $R / \sqrt{2}<r<R$ (or the 
same with the opposite sign, depending on the direction of the magnetic-field sweep). This distribution provides overall neutrality of the sample, while maximum possible charge is moved. The electric field created by this charge distribution in the sample plane can be expressed in terms of elliptic integrals. It is plotted against $r / R$ in Fig. 2 (dashed line). Non-physical negative values of the electric field near the edge indicate that more charge can be placed in this region. Indeed, placing an additional charge density $-e \delta n=-e \Delta n_{c}(T)[\sqrt{b /(R-r)}-1]$ into a narrow strip of width $b \approx 0.02 R$ along the edge eliminates the singularity and makes the field in this region close to zero, in accordance with condition (ii) (solid line in Fig. 2). Further refinement

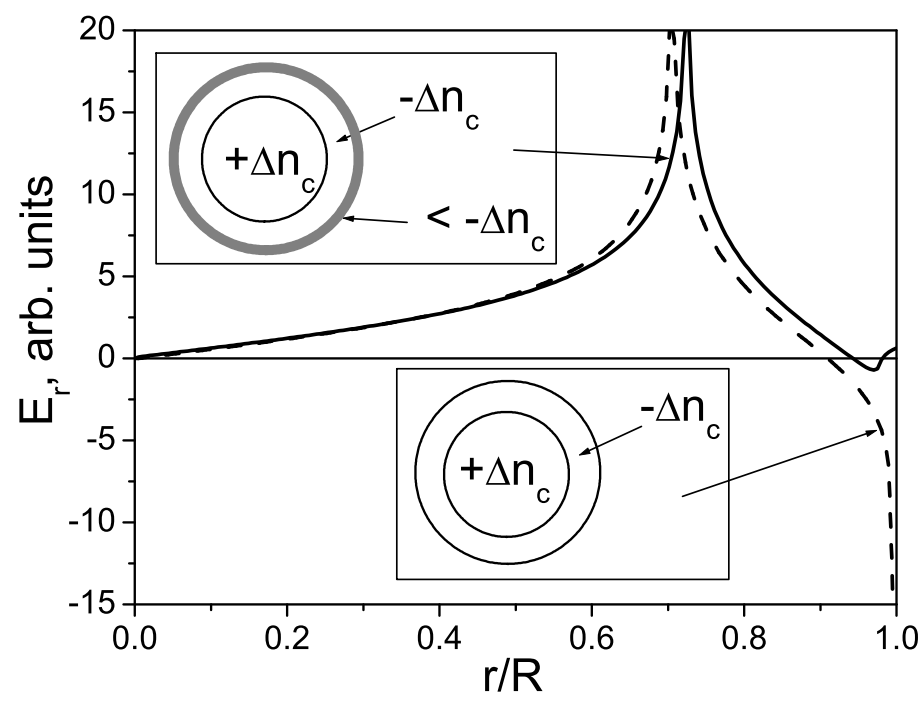

Figure 2: Charge (insets) and radial electric field distribution across the sample.

of the dependence of $E_{r}$ on $r$ is possible, but is not needed, since the contribution of the narrow edge region to the magnetic moment (Eq. (1)) is negligible. As the charge density over all the sample, and therefore $E_{r}$, is proportional to $\Delta n_{c}(T)$, the saturation value of the magnetic moment $M_{S}$, according to Eq.(1), is

$$
M_{S}=\Lambda \frac{\pi}{c} \sigma_{x y} e \Delta n_{c}(T) R^{3}
$$

where $\Lambda$ is the value of the integral in Eq. (1) expressed in dimensionless variables; our numerical calculations give $\Lambda \approx 1.1$. Thus, the temperature dependence of $M_{S}$ is given by Eq. (3) for $\Delta n_{c}(T)$. According to Eq. (3), $\Delta n_{c}(T)$ is the number of localised electron states within the energy range from zero (half-way between the filled and empty levels) to $\varepsilon_{0} / 2+k_{B} T \ln C$ (note that $\ln C$ is negative). $\Delta n_{c}(T)$ vanishes at temperature $T_{c}=\varepsilon_{0} / 2 k_{B}|\ln C|$. As $T \rightarrow 0, \Delta n_{c}(T)$ is one half of the total density of localised states in between the Landau levels. The behaviour of $\Delta n_{c}$ at $T<T_{c}$ 
is derived from the specific energy dependence of the density of states. Notably, if $\rho$ is approximately constant everywhere excepting the vicinity of LLs, as expected for samples with strong disorder, the temperature dependence of $M_{S}$ is linear:

$$
M_{S}=\Lambda \frac{\pi}{c} \sigma_{x y} e R^{3}\left(\varepsilon_{0} / 2-k_{B} T|\ln C|\right) \rho
$$

in agreement with experimental results for 'dirty' samples.

One can see from Eq. (5) that $M_{S}$ is proportional to the density of localised states. Therefore, the more disordered the sample, the higher the current densities it can sustain. However, the radial electric field will eventually reach values comparable to the QUILLS critical field, and this new mechanism will prevent $M_{S}$ from growing further. This should result in saturation of $M_{S}$ as a function of temperature when $T$ approaches zero. This effect is indeed observed. The QUILLS conditions should first be met near $r=R / \sqrt{2}$, where the electric field is the highest. The critical fields

calculated from our experimental data under this assumption agree with the previous theories of QUILLS.

\section{References}

[1] G. Nachtwei, Physica E 4, 79 (1999) and references therein.

[2] L. Eaves and F. W. Shread, Semicond. Sci. Technol. 1, 346 (1986).

[3] J.P. Watts, A. Usher, A.J. Matthews, M. Zhu, M. Elliot, W.G.Herrenden-Harker, P.R. Morris, M.Y. Simmons and D.A. Ritchie, Phys. Rev. Letters 81, 4220 (1998); A.J. Matthews, J.P. Watts, M. Zhu, A. Usher, M. Elliot, W.G.Herrenden-Harker, P.R. Morris, M.Y. Simmons and D.A. Ritchie, Physica E 6, 140 (2000).

[4] M.I. Dyakonov, Solid State Commun. 78, 817 (1991). 